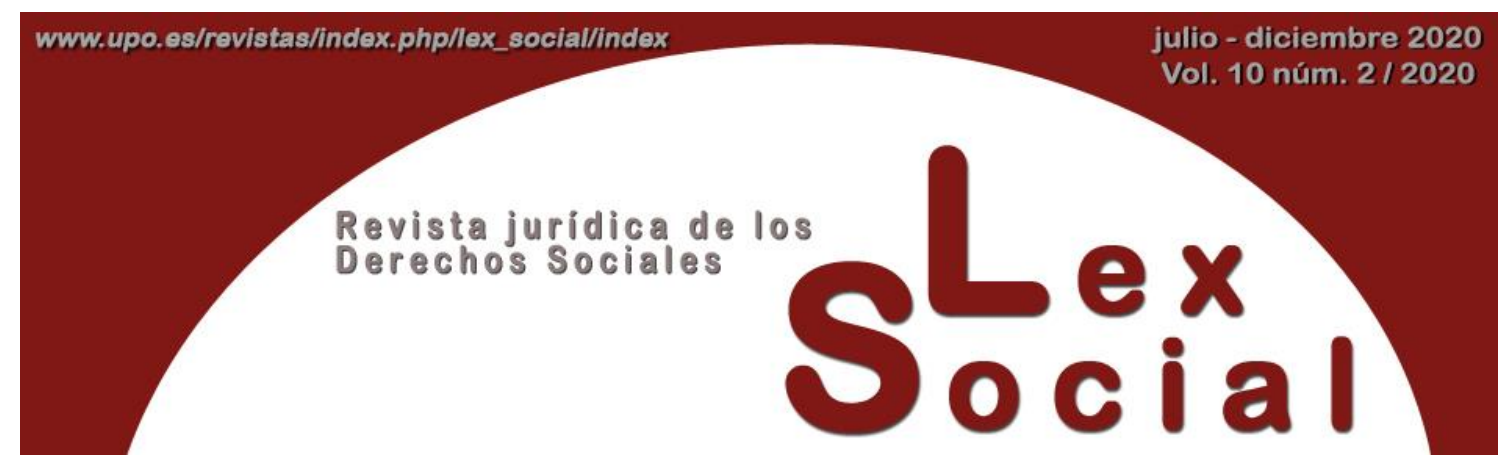

\title{
LAVORO DECENTE E RESPONSABILITÀ DELLE IMPRESE MULTINAZIONALI PER LE PRODUZIONI DELOCALIZZATE: PANORAMA DELLA LEGISLAZIONE ITALIANA
}

\section{DECENT WORK AND RESPONSIBILITY OF MULTINATIONAL COMPANIES FOR DELOCALIZED PRODUCTIONS: AN OVERVIEW OF ITALIAN LEGISLATION}

VINCENZO FERRANTE

Catedrático de Derecho del Trabajo

Universidad Católica de Milán

Artículo recibido el 9 de mayo de 2020

Artículo aceptado el 2 de junio de 2020

DOI: https://doi.org/10.46661/lexsocial.5070

\section{RIASSUNTO}

Il saggio, che prende spunto dal Convegno organizzato dall'Università di Alcalà nel dicembre del 2019 "Estándares laborales y responsabilidad de las empresas multinacionales. Desafíos en un mundo global", esamina la legislazione italiana diretta a garantire che le imprese multinazionali aventi sede nello Stato realizzino scambi commerciali su una base equa, vigilando sulle società controllate estere, per assicurare che queste rispettino i core labour standards previsti dall'OIL e dalle altre organizzazioni internazionali.

A differenza della legislazione di altri paesi europei, manca in Italia una norma che imponga un obiettivo siffatto, forse a ragione del fatto che la vasta diffusione del lavoro sommerso ha imposto al legislatore di concentrarsi sui fenomeni di sfruttamento che si manifestano nei confini nazionali (anche se si rileva come la trasposizione delle direttive che hanno riguardo a questo obiettivo non sempre è correttamente avvenuta).

In attesa che venga a maturare una norma di diritto internazionale universalmente riconosciuta che faccia divieto di commerciare un qualunque bene, quando esso sia 
stato prodotto attraverso lo sfruttamento schiavistico di altri uomini, l'A. si concentra sulla legislazione relativa alle società commerciali, su quella che regola gli appalti privati e sulla disciplina europea del commercio alimentare, per verificare se le norme già esistenti in materia non consentano al giudice nazionale, opportunamente sollecitato, di reprimere lo sfruttamento che avvenga al di fuori dei confini nazionali, in virtù degli obblighi assunti dalle singole società nei confronti dei propri soci e dei consumatori.

Parole Chiave: lavoro dignitoso, giustizia sociale, sviluppo sostenibile, imprese multinazionali, Organizzazione Internazionale del lavoro (ILO/OIT/OIL), commercio internazionale.

\begin{abstract}
The essay, inspired by the conference organized by the University of Alcalà de Henares in December 2019 "Estándares laborales y responsabilidad de las empresas multinacionales. Desafíos en un mundo global", examines the Italian legislation aimed at ensuring that multinational companies based in the State carry out trade on a fair basis, supervising the foreign subsidiaries, to ensure that they comply with the core labor standards established by the ILO and other international organizations.

Unlike the legislation of other European countries, in Italy there are no statutory rules specifically aimed at achieving this result, perhaps because the widespread of undeclared work has forced the legislator to focus on the exploitation phenomena that occur within national borders (even if the A. remarks that the transposition of the European directives on preventing and combating trafficking in human beings and against illegal immigration has not always been correctly done).

Pending the development of a universally recognized rule of international law that prohibits the trading of any good, when it has been produced through the slave labor of other men, the A. focuses on the legislation concerning commercial companies, on provisions regulating private subcontracting and on European rules on food trade, to check if the already existing norms on the matter do not allow the national judge, duly solicited, to repress exploitation that takes place abroad, by virtue of obligations assumed by each company towards its stakeholders and consumers.
\end{abstract}

KEYWORDS: decent work, social justice, Sustainable Development Goals, Multinational enterprises, International Labour Organization, international trade.

\title{
SOMMARIO:
}

I. Commercio internazionale e dumping sociale.

II. Strumenti normativi di contrasto al dumping sociale.

III. Nullità dei negozi traslativi, efficacia dei codici aziendali e forme giuridiche della delocalizzazione.

IV. La legislazione italiana: 
1. La trasposizione della direttiva 2011/36/UE contro la tratta degli esseri umani.

2. La disciplina di contrasto al caporalato mediante la previsione di norme penali.

3. Le norme sulla responsabilità solidale del committente negli appalti.

4. La vigilanza amministrativa sulle qualità dei beni prodotti come strumento di contrasto alla delocalizzazione.

Bibliografia

Annesso

\section{Commercio internazionale e dumping sociale.}

Il termine dumping indica la pratica commerciale che si realizza inondando ${ }^{1}$ un mercato straniero di prodotti venduti a prezzi notevolmente più bassi di quelli adottati per il mercato nazionale. Essa, quindi, attiene al lato dell'offerta e si concretizza in una strategia di penetrazione commerciale. La finalità evidente è quella di conquistare un mercato in breve tempo, per poi poter fissare liberamente il prezzo, dopo aver eliminato la concorrenza. Per questa ragione è spesso necessario un preventivo, segreto, accordo di spartizione del mercato oggetto di speculazione con altri concorrenti, interessati ad operare contemporaneamente nello stesso modo, nello stesso o in un diverso territorio. In questa prospettiva, potrebbe anzi dirsi che questa pratica sta agli scambi commerciali internazionali, come la spartizione di un paese confinante sta alla storia dell'imperialismo.

Il dumping, una volta divenuto palese, può essere contrastato dal paese importatore che lo subisce o attraverso misure intese a stimolare il mercato interno (come barriere doganali, sussidi alle imprese locali, interventi sulle caratteristiche tecniche dei beni esportati, contingentamenti dei prodotti in ingresso), o anche attraverso il ricorso ad altri fornitori stranieri, a condizione, in questo caso, che manchi un monopolio nella produzione del bene offerto sotto-costo.

Esso richiede peraltro, per poter essere efficacemente attuato, una protezione del mercato nazionale, a beneficio dell'impresa esportatrice, verso il rischio di acquisti operati dai clienti direttamente all'estero, ad un prezzo inferiore a quello praticato sul mercato interno, e coerente con i costi effettivamente sopportati per la produzione (e da qui la definizione tradizionale, di "vendita all'estero di una merce a prezzi inferiori a quelli praticati sul mercato interno", , che descrive però solo un aspetto del fenomeno e che non vale a mettere in evidenza il fine ultimo perseguito dall'impresa esportatrice e dallo Stato che la sostiene).

${ }^{1}$ Il verbo inglese to dump significava originariamente scaricare un carro, rovesciandolo longitudinalmente: v. FLORA, Federico, voce Dumping, in Enciclopedia Italiana, Istituto dell'Enciclopedia italiana, Roma, 1932 in http://www.treccani.it/enciclopedia/dumping \%28Enciclopedia-Italiana\%29/

${ }^{2}$ Voce Dumping, in Enciclopedia Italiana on line, 2020. 
Poiché la pratica è nota agli economisti da più di un secolo, si può dire che tutta la legislazione del commercio internazionale ${ }^{3}$, sia essa contenuta in trattati multilaterali o bilaterali, è finalizzata a contenerne $\mathrm{i}$ rischi ${ }^{4}$, mediante un riequilibrio delle reciproche convenienze che determinano gli stati nazionali allo smantellamento delle barriere doganali 5 .

Alla luce di queste brevi notazioni, appare evidente come diverso sia il significato del termine quando esso viene qualificato dall'aggettivo "sociale": in questo caso, il bene viene venduto sotto-costo nel mercato interno, ottenendo un risparmio sui costi di produzione in ordine alla componente legata al lavoro, grazie al trasferimento di una parte della produzione (o dell'intera filiera) nei paesi dove minore è il livello di reddito medio individuale e, conseguentemente, il costo del lavoro.

Il fenomeno quindi dovrebbe essere più correttamente descritto come de-localizzazione della produzione ${ }^{6}$, di modo che ci sarebbe da dubitare dell'opportunità di utilizzare una stessa parola per fenomeni apparentemente così diversi (conquista del mercato estero, nel primo caso, e del mercato nazionale, nel secondo). Non a caso, nell'ambito del diritto europeo, posto che ogni operazione che concretizzi l'esercizio della libertà di prestazione di servizi o di stabilimento deve considerarsi lecita, l'espressione è sostanzialmente assente nella terminologia legislativa e viene, semmai, utilizzata per individuare possibili forme di concorrenza sleale fra imprese, ove si faccia ricorso a lavoro irregolare o alla violazione di norme in tema fiscale e contributivo ${ }^{7}$.

\footnotetext{
${ }^{3}$ In generale, v. TREBILCOCK, Michael J., TRACHTMAN, Joel, Advanced Introduction to International Trade Law, E. Elgar Publ., 2a ed, UK e USA, 2020; COMBA, Andrea (Dir), Neoliberismo internazionale e Global Economic Governance, 2a ed., Torino, Giappichelli, 2013; MAZZONI, Alberto, MALAGUTI, Maria Chiara), Diritto del commercio internazionale, Torino, Giappichelli, 2019.

${ }^{4}$ L'art. 207 TFUE (art. 133 TCE), nell'enunziare i principi su cui si fonda la politica commerciale comune, afferma che essa "è fondata su principi uniformi" anche in ordine alle politiche di esportazione e «alle misure di protezione commerciale, tra cui quelle da adottarsi nei casi di dumping e di sovvenzioni».

${ }^{5}$ Nell'ambito della scienza economica L. Pasinetti ha suggerito che i vantaggi derivino non tanto dalla c.d. specializzazione produttiva, che può darsi per l'agricoltura o per l'attività estrattiva, ma da un fenomeno di emulazione o di apprendimento, che spinge le imprese dei paesi a più basso reddito medio pro capite a replicare la produzione di beni ad alto contenuto tecnologico, una volta che questi abbiano superato le fasi iniziali di commercializzazione e conoscano ormai una larga diffusione di mercato.

${ }^{6}$ Qualche accenno si legge in LYON-CAEN, Antoine, "A proposito del dumping sociale", nel numero monografico Delocalizzazioni produttive e dumping sociale, Lavoro e diritto, 2011, n o 1, pp. 7-12.

${ }^{7} \mathrm{~V}$. in part. la Risoluzione del Parlamento europeo del 14 settembre 2016 sul dumping sociale nell'Unione europea (2015/2255(INI)) (2018/C 204/13), spec. al punto I "Rafforzare i controlli e il coordinamento tra e da parte degli Stati membri”, là dove si condanna «l'utilizzo, da parte di taluni attori economici, di pratiche illegali quali il lavoro sommerso o di pratiche abusive come il falso lavoro autonomo», lamentando che questo può «portare a gravi distorsioni del mercato, a danno delle imprese che lavorano onestamente, in particolare le $\mathrm{PMI}$ », nonché a danno dei sistemi nazionali di protezione sociale, là dove esso determina il «mancato pagamento dei contributi previdenziali». Sul contrasto al lavoro non dichiarato, rinvio al mio $I l$ contrasto all'economia informale nel diritto internazionale del lavoro, in A tutela della prosperità di tutti. L'Italia e l'Organizzazione Internazionale del Lavoro a cent'anni dalla sua fondazione, Milano, Giuffré FL, 2020, pp. 149-162, nonché, per la disciplina UE, a NUNIN, Roberta, "La piattaforma europea di contrasto al lavoro sommerso", in FERRANTE, Vincenzo (Dir.), Economia informale e strategie di contrasto: a che punto siamo?, Milano, Vita e pensiero, 2019.
} 
Non c'è accordo fra gli studiosi dell'economia internazionale circa le ragioni che si pongono alla base del fenomeno ${ }^{8}$ : per un verso, il costo del lavoro continua a rappresentare una parte importante del costo finale dei beni ${ }^{9}$; d'altro lato, è certo che lo sviluppo del sistema dei trasporti, soprattutto navali, ha ridotto grandemente l'incidenza percentuale di questo elemento sui costi complessivi di produzione (anche a motivo del fatto che fra gli equipaggi, e in genere nel settore del trasporto, numeroso è il personale proveniente dai paesi meno industrializzati). Né si deve dimenticare che lo sviluppo dei sistemi di comunicazione consente in molti casi di delocalizzazione al di fuori dei confini nazionali anche servizi, come quelli informatici, in particolare, ma anche call center e gli uffici contabili ${ }^{10}$.

Non manca chi sottolinea come il dumping sociale sia una sorta di strumento di difesa preventiva, contro l'importazione di merci a basso contenuto tecnologico che discende proprio dall'apertura dei mercati dei paesi più ricchi, accettata da questi per poter ottenere, a condizione di reciprocità, l'accesso ai mercati esteri.

Certamente, poi, alla decisione di delocalizzare non è estraneo, il vantaggio che può derivare sul piano dei rapporti interni all'azienda, in conseguenza dell'alternativa che si propone ai sindacati fra moderazione salariale e rischio di delocalizzazione (eventualmente minacciato anche al paese ospite, nella prospettiva di un abbandono dello stesso a vantaggio di un ulteriore spostamento verso altre destinazioni).

Seppure possa apparire, dunque, come un inevitabile fenomeno di adattamento all'apertura dei mercati alla concorrenza, deve ammettersi che si tratta di una pratica ingannevole nei confronti del mercato, quando, mantenendosi il marchio e la rete di distribuzione originaria, si tende comunque a presentare il bene messo in vendita come frutto di una produzione che viene operata in maniera non differente rispetto al passato e, dunque, con caratteristiche diverse da quelle dichiarate al consumatore.

Il dumping sociale, a differenza del dumping, non giova a chi lo attua se rimane nei termini di una operazione limitata nel tempo, poiché, ove il prezzo di produzione venga, anche lentamente, a riallinearsi a quello praticato in origine nei confini nazionali, la

\footnotetext{
${ }^{8}$ VOLPE, Mario, Globalizzazione e dumping sociale: la prospettiva delle teorie economiche, nel numero monografico Delocalizzazioni produttive e dumping sociale, Lavoro e diritto, 2011, $\mathrm{n}^{\circ} 1$, pp. 45-54. A riguardo v. altresì le considerazioni di PEDERSINI, Roberto, "Globalizzazione e politiche commerciali. Non solo deregolamentazione”, Stato e mercato, 2017, nº 1, pp. 105-120.

${ }^{9}$ Ovviamente il vantaggio competitivo nel commercio internazionale deriva da variabili più complesse del costo del lavoro, che coinvolgono la "qualità" delle risorse umane (e quindi la diffusione dell'istruzione e dei sistemi di formazione professionale), la capacità della pubblica amministrazione di attrarre investimenti la c.d. specializzazione produttiva: v. TREU, Tiziano, Globalizzazione e diritti umani. Le clausole sociali nei trattati commerciali e negli scambi internazionali fra imprese, in Stato e mercato, 2017, $\mathrm{n}^{\circ}$ 1, 13-49, anche con il titolo Globalisation and human rights. Social clauses in commercial treaties and in international relations among enterprises in PERULLI, Adalberto, TREU, Tiziano, Sustainable Development, Global Trade and Social Rights, Alphen aan den Rijn, Kluwer Law International, 2018.

${ }^{10}$ Come è noto alcuni call center di imprese nazionali hanno sede nella vicina Albania, dato che in quel paese la popolazione, potendo ricevere il segnale televisivo senza difficoltà, parla in genere l'italiano come seconda lingua, tanto che una legge impone ora all'operatore di comunicare all'utente il luogo in cui si trova chi risponde alla chiamata (art. 1, co. 243, 1. n. 232 del 2016).
} 
delocalizzazione viene a perdere di utilità. L'interesse dell'impresa che trasferisce all'estero la produzione (per intero o solo in parte) è, dunque, quello di evitare interventi normativi delle autorità locali o il formarsi di sindacati, nella prospettiva di mantenere basso il costo del lavoro, anche a costo di raggiungere questo risultato attraverso il mancato rispetto dei diritti dei lavoratori.

Se osservato dal punto di vista del commercio internazionale, il dumping sociale può essere considerato, quindi, come una "inondazione", non sul lato dell'offerta dei prodotti, ma semmai dell'offerta di lavoro. Infatti, lo spostamento di una produzione determina sempre, in brevissimo tempo, un notevole incremento dell'occupazione, poiché ci si avvantaggia di sbocchi di mercato già consolidati, quale quelli dell'impresa che esternalizza. Comune in questo senso è la vocazione monopolistica nei confronti del mercato dello stato che ospita la produzione, che spiega anche le ragioni per cui, consolidatasi la presenza dell'organizzazione produttiva straniera, l'offerta (in questo caso: di posti di lavoro) può anche avvenire ad un costo inferiore rispetto a quello che sarebbe altrimenti praticato, grazie alla forza del numero dei posti di lavoro in gioco, con un evidente ingiusto vantaggio per l'impresa multinazionale.

Si noti che lo stato ospite potrebbe non essere interessato ad adottare una politica antidumping sociale, poiché questa comporterebbe la necessità di fronteggiare la potenziale riduzione della popolazione attiva, dovendosi creare un numero di posti di lavoro, eguale (e con pari garanzie quanto alla loro stabilità), rispetto a quello che verrebbe a perdersi per effetto delle restrizioni eventualmente adottate. In questo senso, è piuttosto il paese della multinazionale che delocalizza ad essere interessato alle politiche di contrasto.

Al contempo, è evidente come la creazione di occupazione e l'incremento del reddito medio pro capite è utile ad assicurare il consenso necessario alla stipula degli accordi internazionali di commercio, posto che uno scambio limitato alla sola esportazione di materie prime renderebbe democraticamente più instabili i governi che hanno sottoscritto gli accordi, mentre l'incremento dell'occupazione, anche se a condizioni talora disumane, vale a rafforzare i governi dei paesi ospiti.

Per queste ragioni, il solo rimedio che il commercio internazionale conosce avverso il dumping sociale pare essere quello dell'avvicinamento delle condizioni di produzione, non tanto mediante un intervento autoritativo sul livello del salario minimo (ove questo esista), ma soprattutto attraverso una armonizzazione della normativa che attiene alle libertà sindacali, alla parità retributiva fa uomo e donna, alla durata del tempo di lavoro e alle politiche di tutela della salute e sicurezza dei lavoratori.

Si tratta di una garanzia reciproca, che finisce per compiacere sia l'opinione pubblica dello Stato dove ha sede la multinazionale, perché in questo modo si ha un incentivo a che almeno la parte tecnologicamente più avanzata della produzione rimanga nel luogo di origine, sia il paese ospite, che vede allontanarsi le ombre del colonialismo.

È indubbio, infatti, che la delocalizzazione può incrementare il tenore di vita dello Stato ospitante, conducendo a forme di redistribuzione della ricchezza e al sorgere di politiche 
di esportazione in proprio di beni prima prodotti per conto terzi. In questo senso, non è inutile sottolineare come lo sviluppo del commercio internazionale richieda relazioni stabili ed amichevoli fra i paesi interessati (e, probabilmente, anche un'inclinazione verso le forme della democrazia rappresentativa). Tale convergenza, come dimostra la storia del Trattato di Roma (ma anche tante altre vicende recenti), non costituisce tuttavia un naturale portato dello sviluppo dei traffici, ma richiede una specifica azione normativa per potersi realizzare.

\section{Strumenti normativi di contrasto al dumping sociale.}

Il commercio fra gli Stati, dunque, per essere equo e solidale (fair trade) richiederebbe un dialogo fra le preoccupazioni commerciali e quelle sociali, perché tutti possano trarre vantaggio dall'incremento degli scambi. Ed è noto come manchi invece un serio coordinamento fra l'Organizzazione internazionale del lavoro (OIL/ILO) e l'Organizzazione Mondiale per il Commercio (OMC/WTO), poiché ci si rifiuta di adottare un sistema di sanzioni commerciali che tenga anche conto del rispetto dei diritti dei lavoratori. Si finisce così, a fronte del blando sistema di enforcement previsto dalla Costituzione dell'OIL, per esporre ad un deficit di efficacia le previsioni del diritto internazionale del lavoro, in quanto esse, sin dalla loro iniziale formulazione nel Trattato di Versailles del 1919, appaiono strutturalmente collegate alle condizioni economiche di contesto $^{11}$.

Al contrario è proprio in forza del collegamento con lo sviluppo del commercio che il diritto internazionale del lavoro può trarre maggior slancio e vigore, essendo tuttavia costretto, in questa prospettiva, ad abbandonare l'idea di un corpus uniformemente applicato dagli Stati, per concentrarsi su quella sua parte più direttamente correlata alla tutela della dignità individuale, che è contenuta nei cc.dd. core labor standards.

Questo è il significato più profondo della Dichiarazione OIL del 1998 che ha condotto all'individuazione delle otto convenzioni "fondamentali", non tanto per abbandonare la tradizionale via della produzione normativa di regole cogenti, ma per definire invece un

\footnotetext{
${ }^{11}$ Per una panoramica generale delle regole sociali nei trattati di commercio, nella vastissima letteratura, v.: ILO, Social dimension of free trade agreements, International Institute for Labor Studies, Geneva, 2013; TREU, Tiziano, "OIL: un secolo per la Giustizia sociale", in FERRANTE, Vincenzo, A tutela della prosperità di tutti. L'Italia e l'Organizzazione Internazionale del Lavoro a cent'anni dalla sua fondazione, Milano, Giuffré FL, 2020, pp. 3-25; PERULLI, Adalberto, "Diritti sociali e commercio internazionale: riflessioni in occasione di un centenario", in FERRANTE, Vincenzo, A tutela della prosperità di tutti. L'Italia e l'Organizzazione Internazionale del Lavoro a cent'anni dalla sua fondazione, Milano, Giuffré FL, 2020, pp. 65-82 e dello stesso A. v. pure PERULLI, Adalberto, "The perspective of social clauses in International Trade", Rapporto al Congresso ISLSSL di Torino, ora in G. CASALE, T. TREU (eds.), Trasformation at work: challenges for the national systems of labour law and social security, Torino, Giappichelli, 2018; GIL Y GIL, José Luis (Dir.), Trade and Labour Standards. New Trends and Challenges, Cambridge Scholars Publishing, ADAPT Labour Studies Book-Series, 2018.
} 
nucleo, sicuramente rilevante ai fini dei costi di produzione ${ }^{12}$, che ambirebbe a trovare applicazione quale parte di uno jus gentium, capace di imporsi in ogni contesto produttivo e dunque quale pre-condizione per l'avvio di negoziati internazionali (e altresì come condizione per il mantenimento dei rapporti commerciali) ${ }^{13}$.

In questo senso, all'obiezione che si sia voluto focalizzare l'attenzione su un nucleo di diritti troppo ristretto, in quanto esclude altri standard che più direttamente venivano a toccare la condizione dei lavoratori dei paesi più sviluppati ${ }^{14}$, si deve rispondere proprio facendo notare che è solo attraverso quest'opera di selezione che si può realizzare più facilmente l'obiettivo di incrementare l'effettività degli standard internazionali, sia che si tratti (i) di rafforzare i meccanismi di monitoraggio, interni all'Organizzazione, sia che si voglia (ii) percorre la via unilaterale del riconoscimento della clausola di nazione più favorita, attraverso il sistema generalizzato di preferenze ${ }^{15}$, sia che infine si intenda (iii) promuovere il rispetto dei diritti dei lavoratori dei paesi terzi attraverso iniziative unilaterali delle singole imprese, quali in primis l'adozione di pratiche di CSR o di contrattazione transnazionale, ovvero adottando strumenti di regolazione soft, sulla scorta di previsioni quali quelle delle Guidelines dell'OCSE o del Global Compact messo a punto dall' $\mathrm{ONU}{ }^{16}$.

\footnotetext{
${ }^{12}$ Restano, invece, escluse ad es. le convenzioni in tema di salute e sicurezza sul lavoro (C155) e sulle ispezioni sul lavoro (C81 e C129), che pure garantirebbero un rilevante strumento di convergenza e che sono richiamate da successivi documenti OIL (come quello sulla "Globalizzazione giusta" del 2008).

${ }^{13}$ Sul punto, v. le considerazioni di GIL Y GIL, José Luis, "Globalización y universalidad del derecho: la lex mercatoria y el derecho internacional del trabajo en el mercado global", Revista Internacional y Comparada de Relaciones Laborales y Derecho del Empleo, 2016, n 2, pp. 18 ss.; GIL Y GIL, José Luis, "La dimensión social de la globalización en los instrumentos de la OIT", Revista Internacional y Comparada de Relaciones Laborales y Derecho del Empleo, 2017, vol. 5, n 1, pp. 12 ss, y GIL Y GIL, José Luis, "Los principios y derechos fundamentales en el trabajo como orden público social universal", in AA.VV., El futuro del trabajo que queremos, Volumen II, "Conversación IV. La gobernanza del trabajo, Conferencia Nacional Tripartita, 28 de marzo de 2017, Palacio de Zurbano, Madrid, Iniciativa del Centenario de la OIT (1919-2019), Madrid, Organización Internacional del Trabajo y Ministerio de Empleo y Seguridad Social, 2017, pp. 503-518.

${ }^{14}$ ALSTON, Philip, "'Core labour standards' and the Transformation of the International Labour Rights Regime", European Journal of International Law, 2004, pp. 457 ss; ALSTON, Philip, Facing Up to the Complexities of the Ilo's Core Labour Standards, European Journal of International Law, 2005, pp. 467 ss., v. MAUPAIN, Francis, "Revitalization Not Retreat: The Real Potential of the 1998 Ilo Declaration of the Universal Protection of Workers' Rights", European Journal of International Law, 2005, p. 439; LANGILLE, Brian A., "Core Labour Rights. The True Story (Reply to Alston)", European Journal of International Law, 2005, pp. 409 ss.; HEPPLE, Bob, "New Approaches to International Labour Regulation", Industrial Law Journal, 1997, pp. 353 ss., pp. 353; WEISS, Manfred, "International Labour Standards: a complex Public-Private Policy Mix", in Studi in onore di Tiziano Treu, Lavoro istituzioni, cambiamento sociale, Napoli, Jovene, 2011, pp. 39-48.

${ }^{15}$ COMPA, Lance, "Labor Rights in the Generalized System of Preferences: A 20-Year Review", Compative Labor Law \& Politicy Journal, 2001, vol. 22, pp. 199-238; SALOMONE, Riccardo, "Preferenze tariffarie generalizzate e core labour standards", nel numero monografico Delocalizzazioni produttive e dumping sociale, Lavoro e diritto, 2011, n 1, pp. 105-118; a riguardo v. il reg. UE n. 978/2012 del 25 ottobre 2012.

${ }^{16}$ TREU, Tiziano, Globalizzazione e diritti umani, cit., 31; nello stesso senso, v. PERULLI, Adalberto, "Globalizzazione e dumping sociale: quali rimedi?", in nel numero monografico Delocalizzazioni produttive e dumping sociale, Lavoro e diritto, 2011, $\mathrm{n}^{\circ} 1$, pp. 13-44.
} 
Fondamentale a riguardo, invero, resta la Dichiarazione tripartita dell'OIL sui principi concernenti le imprese multinazionali ${ }^{17}$, che, come è stato notato ${ }^{18}$, si colloca esattamente alla base della stessa politica di focalizzazione su un cuore di diritti fondamentali.

Le ultime ipotesi, che si sono richiamate sub (iii) e solitamente ricondotte alla formula della "clausola sociale"19, aspirano ad avere il carattere della concretezza, poiché l'effettività delle norme di protezione viene ad essere assicurata grazie agli impegni unilateralmente assunti direttamente dalle imprese, mediante verifiche di conformità (compliance) sulle controllate estere. L'esempio che viene portato a riferimento in questo settore è il diffondersi di protocolli idonei a garantire la sostenibilità della produzione, con l'attribuzione di etichette di qualità e di controllo lungo tutta la "filiera" produttiva, onde evitare il lavoro "schiavistico" 20 . Quest'ultima ipotesi, peraltro, sembra consentire, secondo qualche isolata esperienza, che siano i giudici stessi a dare attuazione agli standard attraverso soluzioni capaci di attivare la giurisdizione nazionale a tutela dei lavoratori stranieri ${ }^{21}$.

In questo senso, la nota pronunzia di un organo internazionale a tutela delle restrizioni introdotte negli USA per la vendita di tonno pescato senza compromettere altre specie

${ }^{17}$ La Tripartite Declaration of Principles concerning Multinational Enterprises and Social Policy è stata adottata dal Consiglio di amministrazione dell'Ufficio internazionale del Lavoro alla 204 ${ }^{\text {a }}$ sessione (Ginevra, novembre 1977), ed emendata dal Consiglio alle 279 (novembre 2000), 295 (marzo 2006) e $329^{a}$ (marzo 2017) sessioni; di essa esiste una traduzione curata dallo stesso BIT con il titolo "Dichiarazione tripartita di principi sulle imprese multinazionali e la politica sociale”, facilmente rintracciabile sul web.

18 A riguardo, v. GÜNTER, Hans, BAILEY, Paul, The Tripartite Declaration of Principles concerning Multinational Enterprises and Social Policy, International Encyclopedia of Labour Law, Boston, Deventer, 1992; ZANOBETTI, Alessandra, Diritto internazionale del lavoro, Milano, Giuffrè, 2011; PERULLI, Adalberto, BRINO, Vania, Manuale di diritto internazionale del lavoro, $2^{\mathrm{a}}$ ed., Torino, Giappichelli, 2015, pp. 123 ss.; GIL Y GIL, José Luis, "Globalización y universalidad del derecho: la lex mercatoria y el derecho internacional del trabajo en el mercado global", op. cit., pp. 24 ss., e "La dimensión social de la globalización en los instrumentos de la OIT", op. cit., pp. 30 ss.

${ }^{19}$ A riguardo, senza pretesa di completezza, v. VAN ROOZENDAAL, Gerda, Trade Unions and Global Governance. The Debate on a Social Clause, London \& N.Y., Continuum, 2002; VANDAELE, Arne, DANIEL, Albert, International labour rights and the social clause: friends or foes, London, Cameron May, 2005; G. AltinTZIS e E. BUSSER, The Lesson from trade agreements for just transitional policies, in Int. Jour. Lab. Research, 2014, 6, pp. 270-294; BRETON, Caroline, Traités de commerce et actes unilatéraux, in THOUVENIN, Jean-Marc, TREBILCOCK, Anne (Dir.), Droit international social: droits économiques, sociaux et culturels, Bruxelles-Paris, Bruylant-CEDIN, 2013; GIL Y GIL, José Luis (Dir.), España y la OIT: 100 años de diálogo en un mundo cambiante, Madrid, Ediciones Cinca, 2017, pp. 203-220, pp. 203 ss.; nella letteratura italiana, v. PERULLI, Adalberto, voce "Lavoro e commercio internazionale", in Digesto delle discipline privatistiche, Aggiornamento, 2000, pp. 444-471; PERONE, Gian Carlo, "Globalizzazione e diritto del lavoro", Il Diritto del lavoro, 2001, pp. 389 ss.; da ultimo FAIOLI, Michele, "Libero scambio, tutele e sostenibilità. Su cosa il TTIP interroga il (nuovo) diritto del lavoro?", Rivista Giuridica del Lavoro, 2015, $\mathrm{n}^{\circ}$ 2, pp. 781 ss.; BRINO, Vania, GRAGNOLI, Enrico, Le imprese multinazionali e il rapporto di lavoro, nel numero monografico di Rivista Giuridica del Lavoro, 2018, $\mathrm{n}^{\circ}$ 2, pp. 209-219.

${ }^{20}$ A riguardo, AA.VV., Impresa, mercato e lavoro schiavistico: alla ricerca di regole efficaci, (a cura del "Centro nazionale di prevenzione e difesa sociale" e dell'Osservatorio "G. Dell'Amore"), Giuffrè, Milano, 2019; CANTONI, Silvia, Lavoro forzato e "nuove schiavitù" nel diritto internazionale, Torino, Giappichelli, 2018.

${ }^{21}$ V. gli esempi citt. in TREU, Tiziano, Globalizzazione e diritti umani, cit., 35-36 
marine ${ }^{22}$ costituisce un esempio che deve far riflettere sulle possibilità dell'ordinamento nel suo complesso di tutelare la vita, non solo dei mammiferi in pericolo di estinzione, ma di tutti gli uomini.

Nulla ovviamente impedisce che una clausola sociale sia imposta in virtù o delle norme delle convenzioni internazionali, che fanno sì che la tutela di popolazioni lontane divenga un obiettivo della legislazione interna (secondo la formula posta a base della dichiarazione di Filadelfia, per cui «poverty anywhere constitutes a danger to prosperity everywhere ${ }^{23}$ ), o attraverso un intervento diretto della legislazione nazionale dei singoli stati, diretto ad imporre obblighi di vigilanza sia sulle imprese che delocalizzano sia su quelle che, più semplicemente, si limitano ad importare prodotti che successivamente vengono immessi in commercio o vengono utilizzati come componenti della produzione di altri beni.

Quest'ultima ipotesi è esattamente quella presa in considerazione dal presente scritto, nella prospettiva di illustrare la legislazione italiana sul punto. Un'ultima riflessione si impone, però, prima di passare all'analisi del dato positivo nei paragrafi da 4 a 7).

\section{Nullità dei negozi traslativi, efficacia dei codici aziendali e forme giuridiche della delocalizzazione.}

Il fenomeno giuridico che richiede di essere normato rappresenta - lo si nota senza malizia - una forma intermedia di maturazione di un nomos che fa fatica a trovare compimento negli scambi economici di ogni giorno: l'idea del rispetto del pianeta (o del creato, se vogliamo utilizzare una definizione teologica) porta taluni a rifiutare oggetti prodotti attraverso un uso dissennato delle ricchezze naturali. Non si deve giudicare questa posizione come illogica, in quanto essa rappresenta in certo modo la coerente conclusione logica della nullità, quale sanzione dell'ordinamento privatistico, diretta a fulminare i negozi giuridici che hanno avuto ad oggetto un bene, venuto ad esistenza in violazione delle norme etiche prese a riferimento.

Invero, quando la nullità discende dalle caratteristiche intrinseche del bene (si pensi per fare un riferimento, noto a tutti e condiviso, ai beni demaniali o alle parti del corpo umano

\footnotetext{
${ }^{22}$ V. BARONCINI, Elisa, 'Il Sistema di etichettatura 'dolphin-safe' e l'Organo d'Appello dell'OMC: la corretta informazione del consumatore e la salute e il benessere degli animali al vaglio del Sistema multilaterale degli scambi", Diritto del commercio internazionale, 2014, pp. 109-134, in https://cris.unibo.it/handle/11585/261491\#.XsZx00QzaUk, che si sofferma sull'utilizzo dell'art. XX GATT nell'ambito dei rapporti commerciali fra Messico e USA. Altri esempi relativi alle corti domestiche in FAIOLI, Michele, "The Quest for a New Generation of Labor Chapter in the TTIP", Economia \& Lavoro, 2015, vol. 49, no 2, pp. 103-120.

${ }^{23}$ In questo modo la condizione interna di ogni stato membro dell'OIL costituisce materia di interesse comune, venendo a configurare il contenuto delle convenzioni come obbligazioni che trascendono lo stretto spazio dei rapporti fra gli Stati per assumere un'efficacia erga omnes: a riguardo, v. DECAUX, Emmanuel, Les formes contemporaines de l'esclavage, Leiden, Nijhoff, 2009 (traggo la citaz. dalla recensione al volume curata da ALLAIN, Jean, Recensione al volume di E. Decaux, Les formes contemporaines de l'esclavage, Nijhoff, Leiden, 2009, European Journal of International Law, 2011, vol. 22, nº 1, pp. 284287.
} 
non suscettibili di essere separate senza comprometterne l'integrità: art. 5 cod. civ. italiano) appare evidente come la conseguenza di tale nullità sia l'assoluta improduttività di effetti di ogni negozio traslativo del bene.

Né pare difficile, alla luce delle previsioni costituzionali e delle Carte internazionali, affermare un concetto di proprietà, non più legato allo jus utendi atque abutendi dell'antica tradizione latina, ma capace di tenere conto della destinazione naturale dei beni e della loro utilità sociale: si tratta, in certo modo, di una forma di (ri)sacralizzazione del rapporto con le cose, ben nota alla cultura delle società rurali del passato, ed oramai in via di estinzione nella moderna società "dei consumi" (con definizione che bene descrive il modificarsi di questo rapporto).

Lo studio comparato, del resto, insegna bene come anche la stessa proprietà dei suoli si presenti in forme storicamente diverse ${ }^{24}$, né mancano disposizioni positive che limitino il potere individuale di abbandonare certi beni di cui si è proprietari, come nei casi degli stessi alimenti ${ }^{25}$, dei rifiuti speciali ${ }^{26}$, del pescato di dimensioni troppo ridotte per essere introdotto in commercio ${ }^{27}$, o di tante altre res, cui la pietà o il rispetto per l'essere umano attribuiscono un valore particolare (e che la bioetica ben conosce).

Si tratterebbe ora di estendere questa intima repulsione, bandendo dal commercio non solo la schiavitù, ma anche $\mathrm{i}$ beni che siano prodotti attraverso l'uso schiavistico del lavoro umano. Si tratterebbe di trasfondere in norme cogenti un imperativo etico che promana dai comportamenti individuali orientati non solo alla conservazione dell'ambiente, ma prima ancora al rispetto della persona umana ${ }^{28}$.

A tale risultato, in assenza di una norma espressa che disponga in tal senso nelle fonti internazionali ${ }^{29}$, può giungersi per quelle stesse strade che poco più sopra si sono analizzate e che ancora coinvolgono le istituzioni nazionali.

\footnotetext{
${ }^{24}$ Rimane ancora vivo il ricordo di BARCELLONA, Mario, "Proprietà privata e forme di merce", Economia e credito, 1977, $\mathrm{n}^{\circ} 4,76$ ss. in part. 34 ss. (dell'estratto).

${ }^{25} \mathrm{La}$ consapevolezza che il diritto al cibo non sia materia solo per i paesi poveri pare oramai assai diffusa; a riguardo V. per tutti THOUVENIN, Jean-Marc, PHILIPPE, Clémence, Le droit à l'alimentation, in THOUVENIN, Jean-Marc, TREBILCOCK, Anne, Droit international social, 2013, 1693-1710; nella letteratura italiana, v. MOSCATELLI, Silvana, Il diritto all'alimentazione nel sistema dei diritti umani, Roma, Aracne, 2014; sul punto v. anche reg. UE n. 223 del 2014, adottato sulla scorta delle disposizioni del TFUE relative alla coesione sociale (art. 175/3) che ha come obiettivo la creazione di un fondo di aiuti e la definizione di programmi operativi (art. 8), d'intesa con le amministrazioni nazionali.

${ }^{26} \mathrm{~V}$. ad es. per i rifiuti elettrici ed elettronici l'art. 227 cod. ambiente (d. lgs. 152/2006), ma gli esempi sarebbero numerosissimi.

${ }^{27}$ Ai fini del ripopolamento biologico, si impone ai pescatori di rilasciare nuovamente in mare quanto non viene comunque avviato al commercio, anche nella prospettiva di favorire l'alimentazione dei pesci.

${ }^{28}$ Il rifiuto di partecipare all'utile che discende dalla commissione di reati finanziari si rintraccia già nella disciplina del riciclaggio (v. C. Giust., 17 gennaio 2018, n. 676, in relazione alla dir. 2015/849), come ricorda anche RESCIGNO, Matteo, "Per una definizione giuridica di impresa schiavistica", in AA. VV. Impresa, mercato e lavoro schiavistico alla ricerca di regole efficaci, (a cura del "Centro nazionale di prevenzione e difesa sociale” e dell'Osservatorio “G. Dell'Amore”), Milano, Giuffrè FL, 2019, p 95.

${ }^{29}$ Mi pare di poter dire che un tale divieto non sia previsto né all'art. 5 Carta di Nizza, né all'art. 4 della CEDU e neanche nella direttiva sulla tratta esseri umani n. 36 del 2001: a commento all'art. 5, v. GRATTERI, Andrea, "Commento all'art. 5", in Aa. Vv., Carta dei diritti fondamentali dell'Unione Europea, Milano, Giuffrè, 2017.
} 
Si tratterebbe - lo si ripete - di prevedere, quindi, una legge che vieti (o comunque limiti) la possibilità di importare e di mettere in commercio beni prodotti con violazione dei CLS, oppure imponga alle società multinazionali uno specifico obbligo di vigilare che l'intera catena produttiva sia rispettosa dei diritti fondamentali dei lavoratori ${ }^{30}, \mathrm{o}$, ancora, attraverso meccanismi, anche giurisprudenziali, diretti a potenziare gli strumenti utili a garantire effettività ai codici etici e agli altri strumenti di responsabilità sociale. Si tratterà in questo caso di predisporre un apparato normativo che consenta di verificare che siano stati messi in vendita solo beni prodotti in conformità agli standard (quali che essi siano) che la stessa impresa ha preventivamente dichiarato di voler rispettare.

In queste direzioni si sono già mosse la legislazione inglese e francese attraverso provvedimenti normativi ${ }^{31}$, mentre in Italia, non sembrano ancora sussistere previsioni di questo tipo, essendosi semmai il legislatore orientato nel senso di ostacolare la delocalizzazione ${ }^{32}$. Allo studioso, quindi, non resta che verificare se non sussistano comunque «strumenti di enforcement diversi da quelli operanti nel sistema dei trattati» ${ }^{33}$ capaci di assicurare il rispetto dei CLS al di fuori dei confini nazionali.

In altri termini, in questa prospettiva, si dovrà cercare di dare il massimo effetto a strumenti di regolazione puramente unilaterali, quali i codici etici o di condotta, adottati dalle imprese, proponendone una lettura che non sia limitata quanto ad ambito territoriale del paese della "casa madre", ma che valga a ricondurre al rispetto dei principi così solennemente enunziati anche fattispecie verificatesi in altri paesi, in quanto comunque appartengono ad una medesima catena produttiva, attraverso società controllate o aziende fornitrici, formalmente autonome, ma di fatto collegate alle multinazionali italiane da legami commerciali stabili.

La riflessione dottrinale a riguardo inizia invero ad essere abbastanza ampia, anche se non sembra che si sia stati in grado di evitare il rischio di un ripiegamento su moduli

\footnotetext{
30 A riguardo, v. LYON-CAEN, Antoine, "Verso un obbligo legale di vigilanza in capo alle imprese multinazionali?" nel numero monografico di Rivista Giuridica del Lavoro, 2018, n 2, pp. 240-249.

${ }^{31} \mathrm{Il}$ caso francese è affrontato in altro saggio del presente numero della rivista. Si veda AUVERGNON, Philippe, "El establecimiento de un deber de vigilancia de las empresas transnacionales, o cómo no dejar que los zorros cuiden libremente del gallinero mundial", Lex Social, julio-diciembre 2020, Vol. 10 núm. 2/2020, pp. 206-223. A riguardo altresì v. LYON-CAEN, Antoine, "Verso un obbligo legale...", cit. a nota che precede; BUCCELLATO, Francesco, "Un modello normativo: il TISC report di cui al Modern Slavery Act del Regno Unito", in AA.VV. Impresa, mercato e lavoro schiavistico: alla ricerca di regole efficaci, Milano, Giuffrè, 2019, pp. 143 ss. Per completezza si deve ricordare che, a mente del considerando n. 38 della dir. 52/2009, la direttiva stessa in tema di repressione del lavoro nero offerto da immigrati irregolari non è applicabile al Regno Unito (già prima della Brexit), a Irlanda e Danimarca.

${ }^{32}$ Si deve però fare menzione dell'art. 5, d.1. 12 luglio 2018, n. 87 (conv. in 1. 9 agosto 2018, n. 96: decreto c.d. "dignità"), secondo cui: «Fatti salvi i vincoli derivanti dai trattati internazionali, le imprese italiane ed estere, operanti nel territorio nazionale, che abbiano beneficiato di un aiuto di Stato che prevede l'effettuazione di investimenti produttivi ai fini dell'attribuzione del beneficio, decadono dal beneficio medesimo qualora l'attività economica interessata dallo stesso o una sua parte venga delocalizzata in Stati non appartenenti all'Unione europea, ad eccezione degli Stati aderenti allo Spazio economico europeo, entro cinque anni dalla data di conclusione dell'iniziativa agevolata».

${ }^{33}$ TREU, Tiziano, Globalizzazione e diritti umani, cit., 31.
} 
sostanzialmente autoreferenziali ${ }^{34}$, incerto restando se ai codici aziendali debba attribuirsi la cogenza propria delle obbligazioni negoziali unilaterali, secondo il modello dell'offerta al pubblico, che coinvolge i consumatori, o quella, forse più efficace, dell'assunzione di una obbligazione unilaterale nei confronti dei lavoratori terzi coinvolti nel ciclo produttivo, riconoscendo quindi ad essi azione nel paese della casa madre per i danni eventualmente derivati dal mancato rispetto delle promesse fatte ${ }^{35}$.

Nella dottrina italiana si è proposto a riguardo di portare a compimento questo sistema, adottando il punto di vista del diritto societario, affermando che non mancherebbero strumenti per considerare alla stregua di un vincolo per gli amministratori queste fonti apparentemente soft, in quanto costituiscono in realtà espressione degli interessi dei soci. $\mathrm{Ne}$ discenderebbe di certo un risultato utile ai fini che si indagano, secondo il modello che impone direttamente in capo agli amministratori e ai vertici societari delle società capogruppo, uno specifico obbligo di vigilanza e controllo per il fatto delle controllate, anche estere. Ed invero, individuata la tutela del lavoro che attiene all'intera filiera produttiva come un obiettivo della società stessa, una siffatta scelta non può che determinare, in estrema conseguenza, l'applicazione dei severi sistemi di repressione propri della disciplina dei mercati regolati, a tutela degli interessi dei $\operatorname{soci}^{36}$.

Lo svantaggio di questo approccio si rintraccia, innanzi tutto, nella necessità che esistano rapporti societari chiari e delineati, poiché un siffatto dovere di influenza potrà affermarsi con maggiore facilità nel caso di controllo della entità estera, mentre esso si indebolisce a fronte di rapporti di semplice fornitura, anche se stabili, poiché il ricorso a contratti che hanno ad oggetto il trasferimento di proprietà viene a collocare in secondo piano le modalità attraverso le quali sono stati prodotti i beni, poi fatti oggetto di scambio.

Non è per caso allora se, per evitare il rischio di facili elusioni, manca una vera e propria definizione di impresa multinazionale nell'ambito degli strumenti internazionali ${ }^{37}$, poiché, al di là della veste giuridica data al rapporto commerciale, è proprio delle imprese multinazionali un controllo attento sulla filiera di produzione delocalizzata, al fine di ottenere un risultato conforme alle caratteristiche richieste, sia che si tratti di prodotti già

\footnotetext{
${ }^{34}$ A riguardo, SCARPONI, Stefania, "Imprese multinazionali e autoregolamentazione transnazionale in materia di lavoro", nel numero monografico di Rivista Giuridica del Lavoro, 2018, no 2, pp. 250-270; BORGIA, Fiammetta, "La soft law come strumento di regolamentazione delle attività delle imprese multinazionali", Diritto del Commercio Internazionale, 2010, vol. 24, nº 2, pp. 309-334 (e già BORGIA, Fiammetta, La responsabilità sociale delle imprese multinazionali, Napoli, Editoriale Scientifica, 2007); in prospettiva comparata, v. le riflessioni di BENATTI, Francesca, "Responsabilità sociale dell'impresa, codici etici, vincoli gestori", in Impresa, mercato e lavoro schiavistico alla ricerca di regole efficaci, (a cura del "Centro nazionale di prevenzione e difesa sociale" e dell'Osservatorio “G. Dell'Amore"), Milano, Giuffrè, 2019, pp. 115-125. DI TURI, Claudio, "Responsabilità sociale delle imprese multinazionali e tutela dei diritti dell'uomo", Rivista Cooperazione Giuridica Internazionale, 2011, no 37, pp. 31-56 (e dello stesso A., Globalizzazione dell'economia e diritti umani fondamentali in materia di lavoro: il ruolo dell'OIL e dell'OMC, Milano, Giuffrè, 2007).

35 ADDANTE, Adriana, "Pratiche commerciali scorrette e forced labour", I Contratti, 2016, n 12, pp. 1135-1146.

${ }^{36}$ V. RESCIGNO, Matteo, op. cit. e già RESCIGNO, Matteo, BUCCELLATO, Francesco (Dir.), Impresa $e$ «forced labour». Strumenti di contrasto, Bologna, Il Mulino, 2015.

${ }^{37}$ GÜNTER, Hans, BAILEY, Paul, op. cit., p. 8 ss.
} 
pronti per essere messi in vendita o, invece, di componenti da assemblare o di prodotti semilavorati.

Il punto, invero, sembra (ma per altri aspetti) ben presente al legislatore italiano, che, al fine di evitare, il consolidarsi di posizioni di vantaggio sul mercato interno, vieta "l'abuso di dipendenza economica" che consegue all'instaurarsi di stabili rapporti commerciali di fornitura di beni o servizi ${ }^{38}$. Ed invero la descrizione di tale fattispecie (battezzata "subfornitura") potrebbe avere un qualche interesse nella prospettiva di definire l'IMN (e potrebbe anche fornire un utile riferimento normativo al giudice nazionale, che volesse farne applicazione per pronunziarsi in merito a contratti che trovano esecuzione al di fuori dei confini nazionali).

Ecco la nozione: «Con il contratto di subfornitura un imprenditore si impegna a effettuare per conto di una impresa committente lavorazioni su prodotti semilavorati o su materie prime forniti dalla committente medesima, o si impegna a fornire all'impresa prodotti o servizi destinati ad essere incorporati o comunque ad essere utilizzati nell'ambito dell'attività economica del committente o nella produzione di un bene complesso, in conformità a progetti esecutivi, conoscenze tecniche e tecnologiche, modelli o prototipi forniti dall'impresa committente». Come si vede è il momento organizzativo che viene in rilievo, descrivendo un ciclo produttivo integrato, nel quale anche i rapporti fra le imprese possono richiedere una particolare tutela.

Un secondo aspetto di debolezza della proposta di implementare i "codici" aziendali attraverso le regole del governo dell'impresa, consapevolmente rilevato ${ }^{39}$, attiene alla incompletezza della legislazione oggi vigente in Italia: perché i meccanismi propri del diritto societario possano attivarsi così da far sorgere un obbligo di vigilanza, non basta che la riprovazione verso i metodi di produzione adottati nelle consociate estere si esprima in termini etici, ma appare necessario che si proceda ad individuare un gruppo di norme e di precetti, il cui mancato rispetto valga a definire in maniera tassativa la nozione di impresa schiavistica. Ed è da quest'ultimo tema che nel paragrafo che segue si inizierà ad esporre più in dettaglio la disciplina positiva italiana diretta a contrastare le forme peggiori di sfruttamento del lavoro.

\section{La legislazione italiana:}

\section{La trasposizione della direttiva $2011 / 36 / \mathrm{UE}$ contro la tratta degli esseri umani.}

Bisogna subito sottolineare come in questa materia non giovi il confronto con la disciplina dell'Unione Europea, non tanto perché questa rappresenti in ogni sua parte un'area economicamente omogena, ma perché tutti i fenomeni di delocalizzazione della produzione che si realizzano in territorio continentale sono chiamati a misurarsi con le

\footnotetext{
38 La legge 18 giugno 1998, n. 192, "Disciplina della subfornitura nelle attività produttive" è stata recentemente parificata, di fatto, quanto alla responsabilità solidale del committente da C. cost. 6 dic. 2017 , n. 254 .

${ }^{39} \mathrm{~V}$. ancora gli AA. cit. a nota 36. 
previsioni dirette a tutelare la libertà di prestazione di servizi, nell'ambito di un mercato concorrenziale.

In questo senso, l'individuazione di un gruppo di norme capace di imporsi alla libera scelta operata dalle parti secondo la disciplina del diritto internazionale privato ${ }^{40}$, viene già operata dalla direttiva 96/71 sul distacco transnazionale, che non a caso ha costituito negli ultimi anni oggetto di numerosi approfondimenti, al fine di evitare fenomeni dove il dumping sociale rischia di essere praticato nell'ambito dei confini nazionali, e non al di fuori di questi, mediante l'applicazione ai rapporti di lavoro posti in essere in forza di distacco transnazionale di una disciplina diversa, da quella del paese che ospita $\mathrm{i}$ lavoratori stranieri ${ }^{41}$.

Un ausilio potrebbe provenire, invero, dalla direttiva del PE e del Consiglio 5 aprile 2011, n. 36 del 5 aprile 2011 «concernente la prevenzione e la repressione della tratta di esseri umani e la protezione delle vittime», che, al fine di scoraggiare la domanda "interna" di tutte quelle prestazioni (illecite) che comportano un grave sfruttamento dell'altrui persona, impone di adottare un quadro normativo uniforme nella materia. In particolare, l'art. 18 (4) stabilisce che «gli Stati membri valutano la possibilità di adottare misure che dispongano che costituisca reato la condotta di chi ricorre consapevolmente ai servizi prestati da una persona che è vittima» di tratta o di sfruttamento.

Sebbene lo strumento europeo richiami nelle considerazioni iniziali (v. nn. 3, 11 e 26) tutte le forme di sfruttamento, menzionando anzi espressamente la convenzione OIL n. 29 del 1930 sul divieto di lavoro forzato, il legislatore italiano, in sede di trasposizione della direttiva, ha concentrato la sua attenzione solo su alcune fattispecie di reato, che attengono alla "riduzione in schiavitù" e alla "tratta", ai reati pedopornografici, alla vendita di organi.

Mi riferisco a un gruppo di norme che si collocano nell'ambito di un capo unitario del codice penale, relativo ai reati "contro la personalità individuale" e nel cui ambito, si rintraccia anche il reato di cui all' art. 603 bis, diretto a reprimere l'intermediazione illecita e lo sfruttamento del lavoro ${ }^{42}$.

\footnotetext{
${ }^{40}$ Per una valutazione più ampia del punto, v. MATTEI, Alberto, SALOMONE, Riccardo, "Conflict of laws e tutela del lavoro nelle imprese multinazionali", nel numero monografico di Rivista Giuridica del Lavoro, 2018, ${ }^{\circ}$ 2, pp. 272-298.

${ }^{41}$ A conferma di questa difficoltà, deve ricordarsi come le norme di applicazione necessaria di cui all'art. 3 della dir. 96/71, relativa al distacco dei lavoratori nell'ambito di una prestazione di servizi, vengano ora ridefinite dall'art. 1 della dir. (UE) 2018/957 del PE e del Consiglio del 28 giugno 2018 destinata ad essere trasposta entro il 30 luglio 2020.

${ }^{42}$ Come introdotto dall'art. 12 del d.1. 13 agosto 2011, n. 138 e riscritto dalla legge 29 ottobre 2016 n. 199 ; a riguardo v. MISCIONE, Michele, "Caporalato e sfruttamento del lavoro", Il Lavoro nella giurisprudenza, 2017, $\mathrm{n}^{\circ}$ 2, 113-118. V., PINTO, Vito, Rapporti lavorativi e legalità in agricoltura. Analisi e proposte, in Giornale di diritto del lavoro e relazioni industriali, 2019, p. 9 ss.; D'ONGHIA, Madia, DE MARTINO, Claudio, "Gli strumenti giuslavoristici di contrasto allo sfruttamento del lavoro in agricoltura nella legge $\mathrm{n}$. 199 del 2016: ancora timide risposte a un fenomeno molto più complesso", in WP C.S.D.L.E. "M. D'Antona", 2018, n ${ }^{\circ}$ 352, in http://csdle.lex.unict.it/docs/workingpapers/Gli-strumenti-giuslavoristici-dicontrasto-allo-sfruttamento-del-lavoro-in-agricoltura-nella-legge-n/5722.aspx; GAROFALO, Domenico, "Il contrasto al fenomeno dello sfruttamento del lavoro (non solo in agricoltura)", Rivista di diritto della
} 
All'inizio la condotta che veniva visualizzata e punita dal legislatore era quella di chi, spesso appartenendo alla stesso gruppo nazionale o etnico dei lavoratori oggetto di sfruttamento, li organizza in squadre e contratta con gli imprenditori o i proprietari terrieri (o con i loro rappresentanti) il tipo di lavoro da fornire e il compenso dovuto. Si tratta, come si comprende, di una specie di cottimo collettivo, dove però manca del tutto un accordo preventivo fra i lavoratori, poiché essi sono tenuti in stato di soggezione da chi li comanda (il "caporale"), generalmente in forza di un potere di intimidazione che si fonda su violenze e ricatti. Una fattispecie, dunque, che poteva senz'altro ricondursi ad altre fattispecie di reato già presenti nel codice penale (come la violenza privata, la minaccia $o$ il sequestro), se non fosse che si intendeva attraverso una previsione specifica segnalare all'opinione pubblica che le istituzioni non rimanevano passivamente a guardare l'emergere di questi fenomeni.

Quanto esteriore fosse la finalità perseguita del legislatore è dimostrato dal fatto che, dopo pochi anni di vigenza (e scarse applicazioni concrete), la norma è stata riformulata nel 2016, in modo che fosse prevista la punibilità anche di chi sfrutta il lavoro altrui, ampliando quindi i confini della fattispecie vietata. Ed invero nel frattempo ci si era resi conto che in molte zone, anche del Nord della Penisola, la condizione dei lavoratori (specie agricoli), anche se cittadini italiani o regolarmente dotati di permesso di soggiorno, sfiora spesso la schiavitù, anche con episodi di morte dovuta all'eccessivo sfruttamento o alle ritorsioni delle organizzazioni criminali che organizzano il bracciantato irregolare.

Tanto è conseguenza, sia della concorrenza di produzioni a basso costo che giungono da altre regioni agricole d'Europa (o del Mondo), sia di operazioni di concentrazioni di mercato, sulle quali probabilmente non si è ancora indagato a sufficienza, che determinano un innaturale abbassamento del prezzo di cessione del prodotto pronto per la vendita. Il risultato è una rincorsa al ribasso del salario orario, che ha determinato lo sviluppo di relazioni industriali alternative a quelle che fanno capo ai sindacati maggiori, con la conseguente sottoscrizione di contratti collettivi, c.d. "pirata", che prevedono minimi orari molto ridotti.

Si tratta in Italia di una prassi del tutto legittima, non solo perché manca una legislazione sui minimi salariali, ma anche perché l'applicazione dei contratti collettivi è lasciata ai rapporti di forza, poiché essi non sono dotati di efficacia erga omnes, malgrado la precisa indicazione in tal senso fornita dal legislatore costituente (art. 39 Cost.).

sicurezza sociale, pp. 229 ss., ora in Scritti in onore di Carlo Cester, 2020; ROTOLO, Giuseppe, "A proposito del "nuovo" delitto di "intermediazione illecita e sfruttamento del lavoro". Note critiche sul controllo penale del c.d. caporalato", in FERRANTE, Vincenzo (Dir.) Economia "informale" e politiche di trasparenza, Milano, Vita e Pensiero, 2017, pp. 149 -163; MONGILLO, Vincenzo, "Il contrasto penale al forced labour: riduzione in schiavitù, caporalato e responsabilità da reato nelle società", in AA.VV. Impresa, mercato e lavoro schiavistico alla ricerca di regole efficaci (a cura del "Centro nazionale di prevenzione e difesa sociale" e dell'Osservatorio "G. Dell'Amore”), Milano, Giuffrè, 2019, pp. 33-60; DE SANTIS, Giovanni, "Caporalato e sfruttamento di lavoro: politiche criminali in tema di protezione del lavoratore. Pregi e limiti dell'attuale disciplina”, Responsabilità civile e previdenza, 2018, nº 5, pp. 17591782 e n⿳0 $^{\circ}$, pp. 2055-2082. 
La norma penale, in questo senso, ha sicuramente contribuito ad arginare il fenomeno descritto, che ha determinato una innaturale crescita del numero complessivo dei contratti collettivi (poco meno di mille), spesso solo nella prospettiva di individuare soluzioni di incerta legittimità, intese principalmente a nascondere una diffusa e radicata presenza di lavoro "nero" (o non dichiarato), senza che gli enti preposti alla vigilanza riescano a reprimere efficacemente il fenomeno ${ }^{43}$.

Attraverso la previsione dell' art. 603 bis ora in esame, invero, non sarebbe neanche troppo difficile per il giudice italiano provare a reprimere fenomeni di lavoro schiavistico che si realizzano nell'ambito di catene di produzione internazionali, poiché il codice penale dispone che essa, al pari delle altre fattispecie introdotte in attuazione della direttiva 2001/36, trovano applicazione anche «quando il fatto è commesso all'estero da cittadino italiano, ovvero in danno di cittadino italiano, ovvero dallo straniero in concorso con cittadino italiano» (art. 604 c.p.).

Tuttavia, come subito si dirà nel paragrafo che segue, non sembra del tutto semplice un'applicazione in questa direzione, non solo per le evidenti limitazioni che l'autorità inquirente incontra nello svolgere indagini al di fuori dei confini nazionali, ma anche a ragione del fatto che gli elementi che dovrebbero tipizzare la fattispecie vietata sono descritti dal legislatore in maniera spesso approssimativa, che non tiene conto né della disciplina internazionale, né, tanto meno, della stessa regolamentazione interna del contratto di lavoro ${ }^{44}$.

Gli eventi qui tratteggiati, in conclusione, forniscono bene l'idea di un certo disallineamento delle preoccupazioni del legislatore italiano rispetto alle ragioni che muovono gli altri stati più industrializzati, poiché sembra completamente mancare la consapevolezza, che si rintraccia facilmente nella direttiva 2011/36/ (che più sopra si è esaminata), ed ancora nella direttiva europea n. 52/2009 rivolta al contrasto dello sfruttamento del lavoro prestato dagli immigrati irregolari, che in Italia ha ricevuto trasposizione frettolosa e quasi certamente incompleta ${ }^{45}$, che alla base del permanere di forme inaccettabili di sfruttamento si colloca la domanda interna di beni e servizi che impongono un ricorso al lavoro sottopagato e reso in condizioni degradanti. Tutte le disposizioni che si sono fino a qui richiamate sono, infatti, frutto di preoccupazioni interne ai confini nazionali, dove l'obiettivo della repressione penale viene considerato alla stregua di un elemento estraneo al tessuto sociale, che richiede di essere espulso dai confini nazionali, mentre nessuna riprovazione sembra doversi mostrare nei confronti di quanti alimentano il mercato dei beni prodotti mediante labor exploitation.

\footnotetext{
${ }^{43}$ Per una sintetica ricostruzione del quadro complessivo, rinvio a FERRANTE, Vincenzo, "Economia "informale" e politiche del lavoro: un nuovo inizio?", in WP CSDLE "M. D'Antona", 2017, n 337, http://csdle.lex.unict.it/docs/workingpapers/Economia-informale-e-politiche-del-lavoro-un-nuovoinizio/5663.aspx

${ }^{44}$ Senza dire che non è nemmeno chiaro ai fini dell'applicazione dell'art. 604 cit. quali standard si devono considerare violati: se quelli nazionali o quelli in vigore nel paese che ospita la produzione.

${ }^{45}$ Dir. 2009/52/CE del PE e del Consiglio del 18 giugno 2009 che introduce norme minime relative a sanzioni e a provvedimenti nei confronti di datori di lavoro che impiegano cittadini di paesi terzi il cui soggiorno è irregolare.
} 


\section{La disciplina di contrasto al "caporalato" mediante la previsione di norme penali.}

La norma di cui all'art. 603 bis c.p., rubricata «intermediazione illecita e sfruttamento del lavoro», prevede una responsabilità penale in capo a chi «recluta manodopera allo scopo di destinarla al lavoro presso terzi in condizioni di sfruttamento, approfittando dello stato di bisogno dei lavoratori», ovvero «utilizza, assume o impiega manodopera ... sottoponendo i lavoratori a condizioni di sfruttamento ed approfittando del loro stato di bisogno».

Si ha sfruttamento, a mente della disposizione ora in esame, quando sussista «(1) la reiterata corresponsione di retribuzioni in modo palesemente difforme dai contratti collettivi nazionali o territoriali stipulati dalle organizzazioni sindacali più rappresentative a livello nazionale, o comunque sproporzionato rispetto alla quantità e qualità del lavoro prestato; (2) la reiterata violazione della normativa relativa all'orario di lavoro, ai periodi di riposo, al riposo settimanale, all'aspettativa obbligatoria, alle ferie; (3) la sussistenza di violazioni delle norme in materia di sicurezza e igiene nei luoghi di lavoro; (4) la sottoposizione del lavoratore a condizioni di lavoro, a metodi di sorveglianza o a situazioni alloggiative degradanti».

Ad esaminare i singoli elementi di fattispecie il risultato è però sconfortante, perché è proprio quest'ultimo richiamo alle condizioni materiali della prestazione che finisce per assumere il maggiore valore qualificativo, anche se è da dire che le condizioni abitative fuoriescono senz'altro dalle obbligazioni che derivano dal contratto di lavoro.

Ed invero, quanto all'elemento del pagamento di una retribuzione "di sussistenza", come già più sopra si è detto, in Italia non sussistono minimi salariali fissati dall'autorità. Né il riferimento ai contratti collettivi è agevole, in assenza di attuazione dell'art. 36 Cost. in tema di equo salario e della seconda parte dell'art. 39 Cost., che dovrebbe garantire efficacia generale (erga omnes) ai contratti collettivi stipulati a livello nazionale secondo il principio maggioritario.

In parole povere e per fare un esempio concreto, poiché in agricoltura - al pari degli altri settori, come sopra si è detto - non c'è un salario minimo, ma sussistono plurimi contratti collettivi, tutti legittimi dal punto di vista del diritto del lavoro, anche se prevedono una retribuzione ben inferiore ai livelli previsti dal contratto collettivo sottoscritto dalle maggiori organizzazioni sindacali, è possibile pagare del tutto legittimamente i lavoratori intorno a 5 euro lordi l'ora. È chiaro che in queste condizioni il lavoro prestato in forma degradante diventa la regola e non l'eccezione.

Un secondo aspetto merita di essere esaminato, in ordine questa volta ai requisiti di cui al punto 2 sopra richiamato: a mente dell'art. 10 d. lgs. n. 66/2003, la domenica non è più un giorno festivo ormai dal 2004. Anzi, bisogna ricordare come i limiti italiani in materia di orario siano quasi disumani, poiché è del tutto lecito (in conformità alla direttiva europea n. 88 del 2003) lavorare 77 ore a settimana per uno svariato numero di settimane di seguito. Poiché la durata massima è limitata solo da un valore medio (48 ore in un periodo di un anno) è consentito, ad es. all'interno del settore alberghiero (ma anche in 
quello agricolo), di lavorare in maniera forsennata per tutta l'alta stagione, purché si recuperi l'attività prestata in più rispetto all'ordinario nei mesi in cui l'albergo è chiuso (senza peraltro alcuna maggiorazione retributiva rispetto ad una prestazione di durata sempre eguale).

Né aiuta il riferimento (di cui al n. 3) alla violazione delle norme in tema di sicurezza e d'igiene, poiché una gran parte delle imprese italiane si trova a violare queste norme, anche se solo per aspetti secondari, cosicché la fattispecie penale, per quanto abbia conseguito un certo successo sul piano fattuale, rischia, perché completamente ignara delle evoluzioni recenti della norma lavoristica, di apparire carente di elementi descrittivi in violazione delle garanzie che discendono dalla previsione costituzionale in tema di riserva di legge (nullum crimen sine lege).

L'esame ora condotto della norma conferma, quindi, che essa è stata formulata senza tenere conto in alcun modo della legislazione, nazionale e interna, in tema di lavoro, con l'evidente rischio che essa sia inutile a definire quella nozione di lavoro schiavistico, indispensabile per un'efficace repressione della delocalizzazione transnazionale. Anzi si deve dubitare financo che essa sia in grado di reprimere i fenomeni che si consumano per intero nei confini patri, se non al prezzo di una evidente e grave forzatura del dato letterale, in violazione del principio di tassatività (e senza che sussistano vere novità rispetto all'applicazione più antica del reato di riduzione in schiavitù di cui all'art. 600 c.p., che pure in passato era stato utilizzato per reprimere le fattispecie ora visualizzate dall'art. 603 c.p. $\left.{ }^{46}\right)$.

\section{Le norme sulla responsabilità solidale del committente negli appalti.}

Nella ricognizione del panorama italiano non si può fare a meno di richiamare qui una norma (art. 29 d. lgs. n. 276/03) che prevede in capo al committente una responsabilità solidale per il pagamento di retribuzioni e contributi dovuti dall'appaltatore, di fatto dando vita ad un fenomeno di c.d. "co-datorialità", poiché il lavoratore è legittimato a rivolgersi direttamente all'impresa committente. La previsione si giustifica soprattutto a mente della larga diffusione di lavoro sottopagato o prestato irregolarmente nei confini nazionali, anche se nulla sembrerebbe impedire che di essa si faccia applicazione anche agli appalti che si svolgono al di fuori dei confini nazionali (sebbene manchino del tutto in giurisprudenza precedenti a riguardo, forse anche a ragione che basta la creazione di una società terza nei paesi presso i quali la produzione si svolge, per inibire l'applicazione della norma, venendosi a configurare così la delocalizzazione come un esempio di vendita di beni fra due distinte società).

La disposizione, fortemente sostenuta dalla maggiore delle organizzazioni sindacali, non prevede un diritto alla parità di trattamento, rispetto a quanti operino alle dirette dipendenze del committente, ma si limita solamente a fornire una garanzia circa il

${ }^{46}$ V. ad es. Cass. pen., sez. V, 19 dic. 2013, n. 3893; Cass. pen., sez. V, 8 febbraio 2013, n. 16313. 
pagamento della retribuzione, in caso di insolvenza dell'impresa appaltatrice che, a tutti gli effetti, resta la sola parte del contratto di lavoro.

La norma, introdotta con l'intento di contrastare fenomeni di elusione diffusi e radicati, contiene quindi una soluzione del tutto indifferenziata, che grava esclusivamente sulle imprese private, sconosciuta al codice (che solo ammetteva una sostanziale azione di surroga ${ }^{47}$ ) e che quasi non riconosce alcun reale rilievo alla genuinità dell'operazione economica realizzata, finendo così per sgravare gli apparati amministrativi dalla soluzione logicamente più piana, che invece dovrebbe puntare al rafforzamento dell'attività pubblica di vigilanza, non solo mediante una intensificazione di questa, ma anche attraverso un suo affinamento, così da renderla più efficace.

$\mathrm{Al}$ tempo stesso la disposizione, incentrandosi solamente sulla responsabilità delle parti, non solamente autorizza una (invero legittima) differenziazione salariale, in relazione alle diverse parti del ciclo produttivo, ma altresì permette l'applicazione di retribuzioni di pura sussistenza da parte dell'appaltatore, a fronte della già richiamate assenza sia della previsione legale di un salario minimo orario, sia di una norma (di attuazione della seconda parte dell'art. 39 Cost.), idonea ad assicurare efficacia generale erga omnes ai contratti collettivi stipulati a livello nazionale dai sindacati maggiormente rappresentativi.

Molto viene sacrificato, quindi, al risultato che si intende raggiungere: non sussiste infatti alcuna norma che imponga al committente di sincerarsi che l'appalto sia rispettoso delle norme in tema di sicurezza dell'ambiente di lavoro ${ }^{48}$ o che le condizioni contrattuali praticate non siano disumane, ma questi è comunque tenuto all'adempimento di tutte le obbligazioni che gravano in capo all'appaltatore nei confronti del personale impiegato. Manca financo una norma che, sul presupposto che sia legittima l'invasione del committente nell'altrui sfera organizzativa, definisca le modalità attraverso le quali può legittimamente svolgersi il controllo quando l'appalto non sia puramente interno (in forma solo documentale? O mediante periodiche ispezioni in loco? Programmate o a sorpresa ? E con o senza un potere di interrogare i dipendenti della società appaltatrice? Ed anche con il coinvolgimento degli enti previdenziali?).

\section{La vigilanza amministrativa sulle qualità dei beni prodotti come strumento di contrasto alla delocalizzazione.}

A fronte di quanto si è venuto sino a qui a dire, appare evidente come la via delle qualità promesse del bene messo in commercio possa in certa misura costituire, almeno in ipotesi specifiche, una strada per far emergere la tutela del lavoro dignitoso. In particolare viene

\footnotetext{
${ }^{47}$ Art. 1676 e v. già art. 1645 c.c. 1865 . La norma è diversa dall'art. 8 della direttiva 52 del 2009, di cui sopra, perché nella direttiva si prevede una responsabilità, ma solo per il ricorso al lavoro irregolare e in relazione, non al committente, ma all'appaltatore e nei confronti dei subappaltatori e dei subappaltatori intermedi.

${ }^{48}$ La questione è trattata ampiamente, sebbene da altro punto di vista, BUCCELLATO, Francesco, Delocalizzazione e "forced labour": un passo indietro nell'azione di contrasto?, Diritto del Commercio Internazionale, 2017, $\mathrm{n}^{\mathrm{o}} 3$, pp. 684-708.
} 
in rilievo di nuovo la qualità del prodotto alimentare e la sua sostenibilità dal punto di vista non solo dell'ecosistema, ma anche del rispetto dei diritti dei lavoratori, secondo il già ricordato sistema di etichettatura sociale, che ha trovato un certo successo fra $\mathrm{i}$ consumatori specialmente con riguardo ai prodotti provenienti dal Sud-America (in particolare caffè e cacao, ma anche cereali e frutta $)^{49}$.

Penso in particolare ad una disciplina, oramai tutta dettata dall'Unione europea, ed in specie all'importante regolamento del PE e del Consiglio n. 625, del 15 marzo 2017, relativo «ai controlli ufficiali ... sugli alimenti e sui mangimi», che si applica anche «alle norme sulla salute e sul benessere degli animali, sulla sanità delle piante nonché sui prodotti fitosanitari». Ancora una volta entrano in gioco le previsioni (v. art. 169 TFUE) in materia di tutela dei consumatori, richiamate nelle premesse ai nn. 2, 3 e 12, anche se formalmente estranee alla base normativa del regolamento (che si fonda sulle competenze dell'Unione in tema di tutela della sanità pubblica, di cui al vicino art. 168 TFUE e in tema di circolazione dei prodotti agricoli).

Un tale rinvio fa rientrare nell'ambito dell'attività di vigilanza esercitata dalle autorità amministrative nazionali anche (v. art. 1, punto 2) «le norme volte a garantire pratiche commerciali leali e a tutelare gli interessi e l'informazione dei consumatori».

In questo modo, attraverso la repressione delle frodi in commercio, si può ipotizzare che vengano a costituire oggetto di controllo da parte delle autorità amministrative anche il mancato rispetto della disciplina che in autonomia l'impresa assegna a sé stessa, mediante i sopra richiamati "codici” aziendali, nei termini di garanzia della qualità del prodotto messo in vendita (o anche solo importato).

Accertato dunque che anche in questo caso si finisce per muoversi nella sfera della tutela dei consumatori ${ }^{50}$, un osservatore smaliziato potrebbe dire che gli interessi verso la tutela dell'ambiente e dell'ecosistema non fuoriescono dalla logica di assicurare la qualità promessa del prodotto in vendita (ed anzi attengono sempre ad un giudizio che si fonda su un diritto reale, quale la proprietà, qui condivisa, delle risolse del globo terrestre), mettendo così sempre in secondo piano la dignità dell'uomo e la sua protezione. L'affermazione potrà anche essere corretta, ma non si vede per quale ragione mai modelli logico-giuridici già sperimentati in altro settore non possano essere adottati al fine di migliorare la vita dei lavoratori dei paesi meno sviluppati.

${ }^{49}$ BONFANTI, Angelica, Imprese multinazionali, diritti umani e ambiente. Profili di diritto internazionale pubblico e privato, Milano, Giuffrè, 2012; ROMANIN JACUR, Francesca, BONFANTI, Angelica, SEATZU, Francesco (Dir.), Natural Resources Grabbing: An International Law Perspective, Leiden, Brill - Nijhoff, 2015; nonché BATTISTI, Anna Maria, Lavoro sostenibile, imperativo per il futuro, Torino, Giappichelli, 2018; TOMASSETTI, Paolo, Diritto del lavoro e ambiente, Milano, Adapt University Press, 2018; CAGNIN, Valentina, Diritto del lavoro e sviluppo sostenibile, Milano, WK CEDAM, 2018.

50 BELLACE, Janice, "Human Rights at Work: The Need for Definitional Coherence in the Global Governance System”, International Journal of Comparative Labor Law and Industrial Relations, 2014, vol. 30, n. 2, pp. 175-198; BELLACE, Janice, The Link Between Trade and Social Clauses, in PERULLI, Adalberto, TREU, Tiziano, Sustainable Development, Global Trade and Social Rights, Alphen aan den Rijn, Kluwer Law International, 2018, pp. 57-69. 
Ci si colloca, ovviamente, ancora nell'ambito di una pura ipotesi, poiché non è dato rinvenire nei repertori o altrimenti notizia di ipotesi nelle quali le istituzioni addette alla vigilanza abbiano potuto accertare che, al di fuori dei confini nazionali, venivano ad essere violati i diritti dei lavoratori coinvolti nella produzione. La norma tuttavia, per l'ampiezza della sua previsione, sembrerebbe pronta a recepire e fare proprie le preoccupazioni che dovessero giungere da organizzazioni no-profit e sindacati, nella prospettiva di provare a sollecitare i giudizi nazionali dei paesi europei, ad una tutela universale delle condizioni di lavoro, a conferma che l'impegno necessario a dare concretezza alle norme internazionali richiede la collaborazione di una platea di soggetti assai ampia e non può essere lasciato solamente nelle mani dell'OIL o degli Stati nazionali.

\section{Bibliografia}

AA.VV., Impresa, mercato e lavoro schiavistico: alla ricerca di regole efficaci, (a cura del "Centro nazionale di prevenzione e difesa sociale" e dell'Osservatorio "G. Dell'Amore”), Giuffrè, Milano, 2019.

ADDANTE, Adriana, "Pratiche commerciali scorrette e forced labour", I Contratti, 2016, no 12 , pp. $1135-1146$.

ALLAIN, Jean, Recensione al volume di E. Decaux, Les formes contemporaines de l'esclavage, Nijhoff, Leiden, 2009, European Journal of International Law, 2011, vol. $22, n^{\circ} 1$, pp. 284-287.

ALSTON, Philip, "'Core labour standards' and the Transformation of the International Labour Rights Regime”, European Journal of International Law, 2004, pp. 457 ss.

ALSTON, Philip, Facing Up to the Complexities of the Ilo's Core Labour Standards, European Journal of International Law, 2005, pp. 467 ss.

AUVERGNON, Philippe, "El establecimiento de un deber de vigilancia de las empresas transnacionales, o cómo no dejar que los zorros cuiden libremente del gallinero mundial", Lex Social, julio-diciembre 2020, Vol. 10 núm. 2/2020, pp. 206-223.

BARCELLONA, Mario, "Proprietà privata e forme di merce", Economia e credito, 1977, $n^{\circ} 4,76$ ss.

BARONCINI, Elisa, 'Il Sistema di etichettatura 'dolphin-safe' e l'Organo d'Appello dell'OMC: la corretta informazione del consumatore e la salute e il benessere degli animali al vaglio del Sistema multilaterale degli scambi”, Diritto del commercio 
internazionale,

2014 ,

pp.

109-134,

in

https://cris.unibo.it/handle/11585/261491\#.XsZx00QzaUk

BATTISTI, Anna Maria, Lavoro sostenibile, imperativo per il futuro, Torino, Giappichelli, 2018.

BELLACE, Janice, "Human Rights at Work: The Need for Definitional Coherence in the Global Governance System", International Journal of Comparative Labor Law and Industrial Relations, 2014, vol. 30, n. 2, pp. 175-198.

BELLACE, Janice, The Link Between Trade and Social Clauses, in PERULLI, Adalberto, TREU, Tiziano, Sustainable Development, Global Trade and Social Rights, Alphen aan den Rijn, Kluwer Law International, 2018, pp. 57-69.

BENATTI, Francesca, "Responsabilità sociale dell'impresa, codici etici, vincoli gestori", in Impresa, mercato e lavoro schiavistico alla ricerca di regole efficaci, (a cura del "Centro nazionale di prevenzione e difesa sociale" e dell'Osservatorio "G. Dell'Amore"), Milano, Giuffrè, 2019, pp. 115-125.

BONFANTI, Angelica, Imprese multinazionali, diritti umani e ambiente. Profili di diritto internazionale pubblico e privato, Milano, Giuffrè, 2012.

BORGIA, Fiammetta, La responsabilità sociale delle imprese multinazionali, Napoli, Editoriale Scientifica, 2007.

BORGIA, Fiammetta, "La soft law come strumento di regolamentazione delle attività delle imprese multinazionali”, Diritto del Commercio Internazionale, 2010, vol. 24, $\mathrm{n}^{\circ} 2$, pp. 309-334.

BRETON, Caroline, Traités de commerce et actes unilatéraux, in THOUVENIN, JeanMarc, TREBILCOCK, Anne (Dir.), Droit international social: droits économiques, sociaux et culturels, Bruxelles-Paris, Bruylant-CEDIN, 2013.

BRINO, Vania, GRAGNOLI, Enrico, Le imprese multinazionali e il rapporto di lavoro, nel numero monografico di Rivista Giuridica del Lavoro, 2018, n 2, pp. 209-219.

BUCCELLATO, Francesco, "Un modello normativo: il TISC report di cui al Modern Slavery Act del Regno Unito", in AA.VV. Impresa, mercato e lavoro schiavistico: alla ricerca di regole efficaci, Milano, Giuffrè, 2019, pp. 143 ss.

BUCCELLATO, Francesco, Delocalizzazione e "forced labour": un passo indietro nell'azione di contrasto?, Diritto del Commercio Internazionale, 2017, nº 3, pp. 684-708. 
CAGNIN, Valentina, Diritto del lavoro e sviluppo sostenibile, Milano, WK CEDAM, 2018.

CANTONI, Silvia, Lavoro forzato e "nuove schiavitù" nel diritto internazionale, Torino, Giappichelli, 2018.

COMBA, Andrea (Dir), Neoliberismo internazionale e Global Economic Governance, $2^{\mathrm{a}}$ ed., Torino, Giappichelli, 2013.

COMPA, Lance, "Labor Rights in the Generalized System of Preferences: A 20-Year Review”, Compative Labor Law \& Politicy Journal, 2001, vol. 22, pp. 199-238.

GAROFALO, Domenico, "Il contrasto al fenomeno dello sfruttamento del lavoro (non solo in agricoltura)", Rivista di diritto della sicurezza sociale, pp. 229 ss.

DE SANTIS, Giovanni, "Caporalato e sfruttamento di lavoro: politiche criminali in tema di protezione del lavoratore. Pregi e limiti dell'attuale disciplina”, Responsabilità civile e previdenza, 2018, n 5, pp. 1759-1782 e nº 6, pp. 2055-2082.

D’ONGHIA, Madia, DE MARTINO, Claudio, “Gli strumenti giuslavoristici di contrasto allo sfruttamento del lavoro in agricoltura nella legge n. 199 del 2016: ancora timide risposte a un fenomeno molto più complesso", in WP C.S.D.L.E. "M. D'Antona”, 2018, $\mathrm{n}^{\mathrm{o}} 352$, in http://csdle.lex.unict.it/docs/workingpapers/Gli-strumenti-giuslavoristici-dicontrasto-allo-sfruttamento-del-lavoro-in-agricoltura-nella-legge-n/5722.aspx

DI TURI, Claudio, Globalizzazione dell'economia e diritti umani fondamentali in materia di lavoro: il ruolo dell'OIL e dell'OMC, Milano, Giuffrè, 2007.

DI TURI, Claudio, "Responsabilità sociale delle imprese multinazionali e tutela dei diritti dell'uomo", Rivista Cooperazione Giuridica Internazionale, 2011, nº 37, pp. 31-56.

DECAUX, Emmanuel, Les formes contemporaines de l'esclavage, Leiden, Nijhoff, 2009.

FAIOLI, Michele, "Libero scambio, tutele e sostenibilità. Su cosa il TTIP interroga il (nuovo) diritto del lavoro?", Rivista Giuridica del Lavoro, 2015, n 2, pp. 781 ss.

FAIOLI, Michele, "The Quest for a New Generation of Labor Chapter in the TTIP", Economia \& Lavoro, 2015, vol. 49, nº 2, pp. 103-120.

FERRANTE, Vincenzo, "Economia "informale" e politiche del lavoro: un nuovo inizio?", in WP CSDLE “M. D'Antona”, 2017, $\mathrm{n}^{\mathrm{o}} 337$, 
http://csdle.lex.unict.it/docs/workingpapers/Economia-informale-e-politiche-del-lavoroun-nuovo-inizio/5663.aspx

FERRANTE, Vincenzo (Dir.), Economia informale e politiche di trasparenza, Milano, Vita e pensiero, 2017.

FERRANTE, Vincenzo (Dir.), Economia informale e strategie di contrasto: a che punto siamo?, Milano, Vita e pensiero, 2019.

FERRANTE, Vincenzo, A tutela della prosperità di tutti. L'Italia e l'Organizzazione Internazionale del Lavoro a cent'anni dalla sua fondazione, Milano, Giuffré FL, 2020.

FLORA, Federico, voce Dumping, in Enciclopedia Italiana, Istituto dell'Enciclopedia italiana, Roma, 1932 in http://www.treccani.it/enciclopedia/dumping \%28EnciclopediaItaliana\%29/.

GIL Y GIL, José Luis, “Globalización y universalidad del derecho: la lex mercatoria y el derecho internacional del trabajo en el mercado global", Revista Internacional y Comparada de Relaciones Laborales y Derecho del Empleo, 2016, n², 46-pp.

GIL Y GIL, José Luis (Dir.), España y la OIT: 100 años de diálogo en un mundo cambiante, Madrid, Ediciones Cinca, 2017.

GIL Y GIL, José Luis, "Los principios y derechos fundamentales en el trabajo como orden público social universal", in AA.VV., El futuro del trabajo que queremos, Volumen II, "Conversación IV. La gobernanza del trabajo, Conferencia Nacional Tripartita, 28 de marzo de 2017, Palacio de Zurbano, Madrid, Iniciativa del Centenario de la OIT (19192019), Madrid, Organización Internacional del Trabajo y Ministerio de Empleo y Seguridad Social, 2017, pp. 503-518.

GIL Y GIL, José Luis, "La dimensión social de la globalización en los instrumentos de la OIT”, Revista Internacional y Comparada de Relaciones Laborales y Derecho del Empleo, 2017, vol. 5, no 1, 54 pp.

GIL Y GIL, José Luis (Dir.), Trade and Labour Standards. New Trends and Challenges, Cambridge Scholars Publishing, ADAPT Labour Studies Book-Series, 2018.

GRATTERI, Andrea, “Commento all'art. 5”, in Aa. Vv., Carta dei diritti fondamentali dell’Unione Europea, Milano, Giuffrè, 2017. 
GÜNTER, Hans, BAILEY, Paul, The Tripartite Declaration of Principles concerning Multinational Enterprises and Social Policy, International Encyclopedia of Labour Law, Boston, Deventer, 1992.

HEPPLE, Bob, "New Approaches to International Labour Regulation", Industrial Law Journal, 1997, pp. 353 ss.

LANGILLE, Brian A., "Core Labour Rights. The True Story (Reply to Alston)", European Journal of International Law, 2005, pp. 409 ss.

LYON-CAEN, Antoine, "A proposito del dumping sociale", nel numero monografico Delocalizzazioni produttive e dumping sociale, Lavoro e diritto, 2011, ${ }^{\circ}{ }^{\circ}$, pp. 7-12.

LYON-CAEN, Antoine, "Verso un obbligo legale di vigilanza in capo alle imprese multinazionali?" nel numero monografico di Rivista Giuridica del Lavoro, 2018, n 2, pp. 240-249.

MATTEI, Alberto, SALOMONE, Riccardo, "Conflict of laws e tutela del lavoro nelle imprese multinazionali”, nel numero monografico di Rivista Giuridica del Lavoro, 2018, $\mathrm{n}^{\circ} 2$, pp. 272-298.

MAUPAIN, Francis, "Revitalization Not Retreat: The Real Potential of the 1998 Ilo Declaration of the Universal Protection of Workers' Rights", European Journal of International Law, 2005, p. 439.

MAZZONI, Alberto, MALAGUTI, Maria Chiara), Diritto del commercio internazionale, Torino, Giappichelli, 2019.

MISCIONE, Michele, "Caporalato e sfruttamento del lavoro", Il Lavoro nella giurisprudenza, 2017, $\mathrm{n}^{\mathrm{o}}$ 2, 113-118.

MONGILLO, Vincenzo, "Il contrasto penale al forced labour: riduzione in schiavitù, caporalato e responsabilità da reato nelle società", in AA.VV. Impresa, mercato e lavoro schiavistico alla ricerca di regole efficaci (a cura del "Centro nazionale di prevenzione e difesa sociale” e dell’Osservatorio “G. Dell'Amore”), Milano, Giuffrè, 2019, pp. 33-60.

MOSCATELLI, Silvana, Il diritto all'alimentazione nel sistema dei diritti umani, Roma, Aracne, 2014.

NUNIN, Roberta, "La piattaforma europea di contrasto al lavoro sommerso", in FERRANTE, Vincenzo (Dir.), Economia informale e strategie di contrasto: a che punto siamo?, Milano, Vita e pensiero, 2019. 
PEDERSINI, Roberto, "Globalizzazione e politiche commerciali. Non solo deregolamentazione", Stato e mercato, 2017, no 1, pp. 105-120.

PERONE, Gian Carlo, "Globalizzazione e diritto del lavoro", Il Diritto del lavoro, 2001, pp. 389 ss.

PERULLI, Adalberto, voce "Lavoro e commercio internazionale", in Digesto delle discipline privatistiche, Aggiornamento, 2000, pp. 444-471.

PERULLI, Adalberto, “Globalizzazione e dumping sociale: quali rimedi?”, in nel numero monografico Delocalizzazioni produttive e dumping sociale, Lavoro e diritto, 2011, $\mathrm{n}^{\mathrm{o}} 1$, pp. 13-44.

PERULLI, Adalberto, "The perspective of social clauses in International Trade", Rapporto al Congresso ISLSSL di Torino, ora in G. CASALE, T. TREU (eds.), Trasformation at work: challenges for the national systems of labour law and social security, Torino, Giappichelli, 2018.

PERULLI, Adalberto, "Diritti sociali e commercio internazionale: riflessioni in occasione di un centenario", in FERRANTE, Vincenzo, A tutela della prosperità di tutti. L'Italia e l'Organizzazione Internazionale del Lavoro a cent'anni dalla sua fondazione, Milano, Giuffré FL, 2020, pp. 65-82.

PERULLI, Adalberto, BRINO, Vania, Manuale di diritto internazionale del lavoro, $2^{\mathrm{a}}$ ed., Torino, Giappichelli, 2015, pp. 123 ss.

PERULli, Adalberto, TREU, Tiziano, Sustainable Development, Global Trade and Social Rights, Alphen aan den Rijn, Kluwer Law International, 2018.

PINTO, Vito, Rapporti lavorativi e legalità in agricoltura. Analisi e proposte, in Giornale di diritto del lavoro e relazioni industriali, 2019, p. 9 ss.

RESCIGNO, Matteo, "Per una definizione giuridica di impresa schiavistica", in AA. VV. Impresa, mercato e lavoro schiavistico alla ricerca di regole efficaci, (a cura del "Centro nazionale di prevenzione e difesa sociale" e dell'Osservatorio “G. Dell'Amore”), Milano, Giuffrè FL, 2019, pp. 93-98.

RESCIGNO, Matteo, BUCCELLATO, Francesco (Dir.), Impresa e «forced labour». Strumenti di contrasto, Bologna, Il Mulino, 2015. 
ROMANIN JACUR, Francesca, BONFANTI, Angelica, SEATZU, Francesco (Dir.), Natural Resources Grabbing: An International Law Perspective, Leiden, Brill - Nijhoff, 2015.

ROTOLO, Giuseppe, "A proposito del "nuovo" delitto di "intermediazione illecita e sfruttamento del lavoro". Note critiche sul controllo penale del c.d. caporalato", in FERRANTE, Vincenzo (Dir.) Economia “informale” e politiche di trasparenza, Milano, Vita e Pensiero, 2017, pp. 149 -163.

SALOMONE, Riccardo, "Preferenze tariffarie generalizzate e core labour standards", nel numero monografico Delocalizzazioni produttive e dumping sociale, Lavoro e diritto, 2011, n ${ }^{\circ} 1$, pp. 105-118.

SCARPONI, Stefania, "Imprese multinazionali e autoregolamentazione transnazionale in materia di lavoro", nel numero monografico di Rivista Giuridica del Lavoro, 2018, n 2 , pp. 250-270.

THOUVENIN, Jean-Marc, PHILIPPE, Clémence, Le droit à l'alimentation, in THOUVENIN, Jean-Marc, TREBILCOCK, Anne, Droit international social, 2013, 1693-1710.

THOUVENIN, Jean-Marc, TREBILCOCK, Anne (Dir.), Droit international social: droits économiques, sociaux et culturels, Bruxelles-Paris, Bruylant-CEDIN, 2013.

TOMASSETTI, Paolo, Diritto del lavoro e ambiente, Milano, Adapt University Press, 2018 .

TREBILCOCK, Michael J., TRACHTMAN, Joel, Advanced Introduction to International Trade Law, E. Elgar Publ., 2a ed, UK e USA, 2020.

TREU, Tiziano, Globalizzazione e diritti umani. Le clausole sociali nei trattati commerciali e negli scambi internazionali fra imprese, in Stato e mercato, 2017, n 1, 1349.

TREU, Tiziano, "OIL: un secolo per la Giustizia sociale", in FERRANTE, Vincenzo, $A$ tutela della prosperità di tutti. L'Italia e l'Organizzazione Internazionale del Lavoro a cent'anni dalla sua fondazione, Milano, Giuffré FL, 2020, pp. 3-25.

VAN ROOZENDAAL, Gerda, Trade Unions and Global Governance. The Debate on a Social Clause, London \& N.Y., Continuum, 2002. 
VANDAELE, Arne, DANIEL, Albert, International labour rights and the social clause: friends or foes, London, Cameron May, 2005.

VOLPE, Mario, Globalizzazione e dumping sociale: la prospettiva delle teorie economiche, nel numero monografico Delocalizzazioni produttive e dumping sociale, Lavoro e diritto, 2011, n 1, pp. 45-54.

WEISS, Manfred, "International Labour Standards: a complex Public-Private Policy Mix", in Studi in onore di Tiziano Treu, Lavoro istituzioni, cambiamento sociale, Napoli, Jovene, 2011, pp. 39-48.

ZANOBETTI, Alessandra, Diritto internazionale del lavoro, Milano, Giuffrè, 2011.

\section{Annesso}

ILO, Social dimension of free trade agreements, International Institute for Labor Studies, Geneva, 2013. 